\title{
Ultrasonics: Some Properties of Inaudible Sound.*
}

\section{By Prof. F. Luoyd Hopwood.}

$\mathrm{T}$ HE intensity or loudness of a sound depends upon the extent or amplitude of the vibration set up, and its pitch upon the frequency or number of vibrations per second. Disturbances of the same type as sound waves may be inaudible either because the intensity (loudness) is insufficient or because the ear is deaf to those particular frequencies : the normal range of hearing of a human being extends approximately from 20 to 20,000 vibrations per second.

'Sounds' of higher frequency than 20,000 are called supersonic or ultrasonic. They can be generated in a variety of simple ways, as, for example, by rubbing a resined rag along a steel wire or by tapping the end of a short rod of material with a suitable hammer ; thus, a steel rod $10 \mathrm{~cm}$. long, clamped at its middle point and tapped at one end, will emit an inaudible 'note' of 26,000 vibrations per second, and when two steel balls 1 in. in diameter collide, they vibrate after impact at the rate of more than 100,000 vibrations per second.

Such ultrasonic emission is discontinuous, of feeble intensity, and is accompanied by parasitic audible sounds. The most convenient generator of sustained vibrations is the valve-oscillator. The vibrator itself is a disc of natural quartz crystal cut with its plane faces parallel to its optic axis and at right angles to an electric or binary axis. Such a disc was shown by MM. Pierre and Jacques Curie to possess the remarkable property that when it is compressed its faces become oppositely electrified, and that its electric charges become reversed in sign if the disc is subjected to extension instead of com. pression. Periodic reversal of stresses is immediately followed by periodic reversal of electrification. Prof. Pierre Curie also showed that natural quartz, in common with certain other crystals, possesses the converse property, namely, that if the opposite faces of a suitably cut disc are electrified, the crystal contracts in one direction and expands in another.

This latter property was utilised by Prof. Langevin of Paris to produce high-frequency sound vibrations by applying alternating electric potentials to electrodes in contact with a quartz plate. The plate vibrates with its maximum amplitude when the frequency of the electrical alternations coincides with a natural frequency of mechanical vibra. tion of the quartz; in other words, when resonance is obtained.

For our experiments we use a quartz crystal cut in the form of a circular disc more than 7 centimetres in diameter and nearly 5 millimetres thick. This is clamped with one face in contact with a massive lead electrode and immersed in transformer oil contained in a small glass tank (Fig. 1). The second electrode, which is made of light copper foil, rests in contact with the other face, and both electrodes are connected to a powerful (3 kilowatt) valve-oscillator, executing half a million vibrations

* From a Friday Evening Discourse delivered at the Royal Institution on Feb. 13. a second, or more. The use of the oil permits the application of very high voltages to the crystal, and also makes it easy to subject small objects to the influence of the very intense vibrations.

\section{Experiments with a Horizontal Beam OF Sound.}

Following Prof. R. W. Boyle, the production of stationary waves in the oil can be rendered visible by the striæ formed in coke dust scattered on a horizontal opal-glass plate placed in the sound beam. The distance between successive striæ, that is, half a wave-length of the sound in oil, is here between $1 \mathrm{~mm}$. and $2 \mathrm{~mm}$. This wave-length is so short in comparison with the dimensions of the crystal and other objects in the tank that these waves exhibit

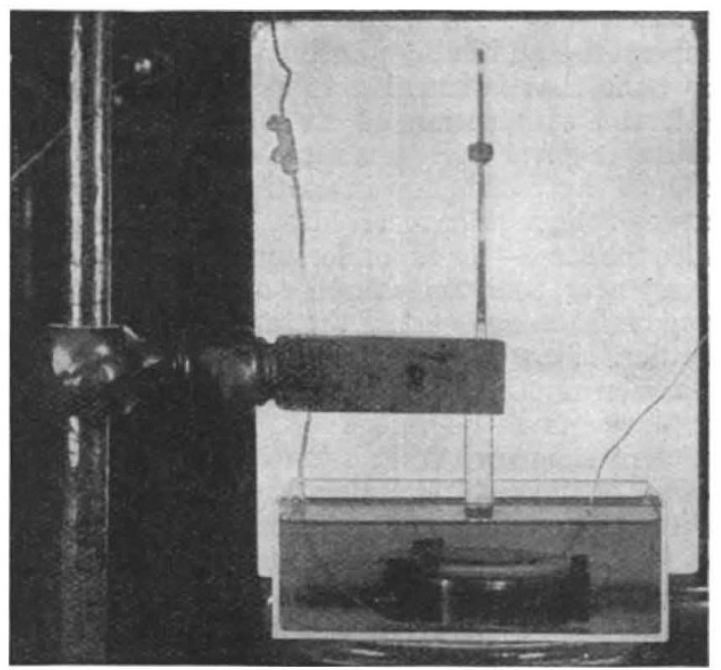

FIG. 1.-Arrangement of quartz crystal and electrodes in oil bath when projecting a vertical beam of sound.

the directional properties ordinarily associated with light waves. Thus, by using plane and curved reflectors, the well-known laws of reflection are verified. In the same way refraction of sound waves by prisms and lenses can be demonstrated. Fig. 2 shows that sound waves travelling through pentane, and falling on a parallel layer of transformer oil, are transmitted if the angle of incidence is less than a certain value (critical angle), but totally reflected if it exceeds this value (total internal reflection). In fact, most objective optical phenomena, except polarisation, can also be faithfully paralleled by these means.

Another application of the coke-dust method of showing stationary sound waves is to the study of architectural acoustics. Fig. 3 shows the "whispering gallery' effect in a model of the lecture theatre of the Royal Institution, using ultrasonic vibrations instead of audible sound. The clinging of the waves to the circular wall is very marked.

The pressure of radiation to which an obstacle is subjected when sound waves fall upon it is shown 
by the rotation of the vane of a torsion pendulum placed in the horizontal ultrasonic beam. This affords a convenient method of estimating the intensity of the sound, which can be used for measur-
Incident Beam.

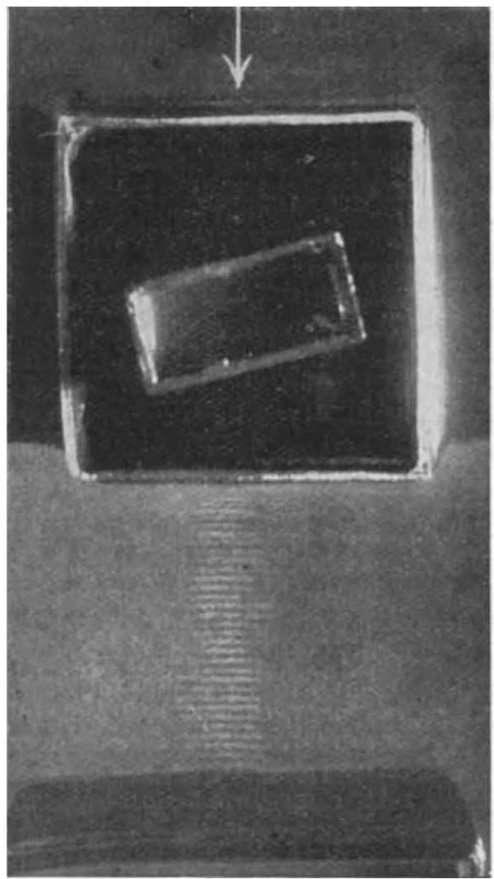

(a)
Incident Beam.

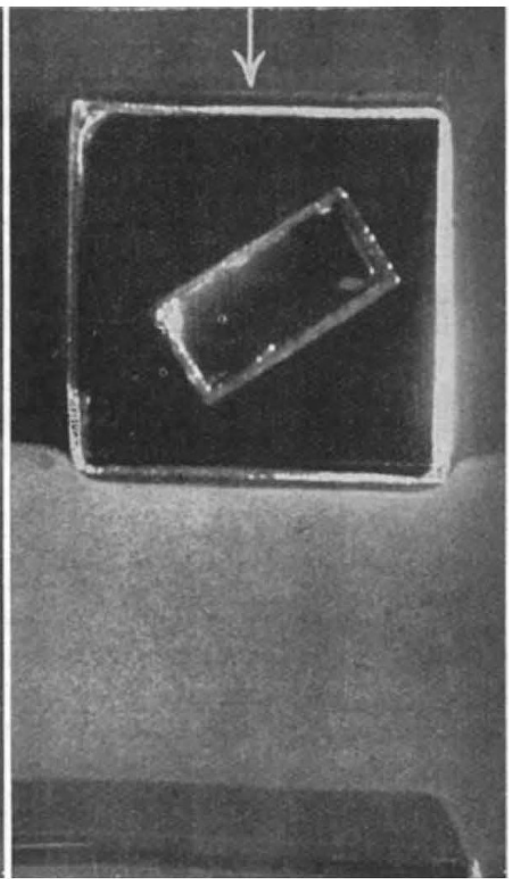

(b)
FIG. 2.-Transmission of ultrasonic waves through layers of pentane and transformer oil for $(a)$ angle of incidence less than critical angle; $(b)$ no transmission for angle of incidence greater than critical angle. The outer vessel contajns pentane and the inner oil.

ing the acoustic reflection and transmission coefficients of different materials.

Experiments With the Ultrasonic Beam DIRECTED TOWARDS THE SURFACE OF A LIqUID.

The properties of ultrasonic waves described above were predicted from theoretical considerations before they were experimentally demonstrated by Langevin, Boyle, and others. Remarkable and hitherto quite unforeseen effects can, however, also be produced by ultrasonic vibrations when these are of great intensity and very high frequency.

With the crystal arranged as shown in Fig. 1, the ultrasonic beam is confined mainly to the column of liquid between the upper crystal face and the surface of the oil. Prof. R. W. Wood and Mr. Loomis have shown that in these circumstances the great concentration of energy causes the amplitude of vibration of the disturbed particles of liquid greatly to exceed the amplitude of vibration of the crystal itself. At the same time the pressure of radiation produces on the surface a mound of oil, several centimetres or even inches in height, which erupts droplets like a miniature fountain. On plunging vessels of appropriate form into this mound of oil, vibrations of great intensity may be communicated to the walls of the vessels, or through the walls to liquids contained in them. If a test-tube contain- ing water is partially immersed in the oil, bubbles of dissolved gas are usually liberated in it (Fig. 4). These behave in a peculiar manner. For moderate intensities of vibration the bubbles form slowly and remain practically stationary at the nodes of the waves formed in the water. As the bubbles grow in size they oscillate and tend to rise to the surface in an irregular zigzag manner as if they were going up a staircase. For still greater intensities we have found that no visible gas bubbles can either exist or be formed. The density of the gas being much less than that of the liquid, the bubbles formed at low intensities must experience the same forces and therefore have greater amplitudes of vibration than the particles of liquid they displace. The result is that at high inten. sities they are deformed and shattered and so dispersed through the liquid as to become and remain effectively in solution.

Cavitation can be produced in liquids such as pentane or benzene, by using a U-tube closed at one end, the closed limb being filled with the liquid, and placed in the mound of oil above the vibrating crystal. The bubbles of vapour formed in the interior of the liquid and collected in the closed limb can be again condensed into liquid by raising the air pressure on the open limb. Using now an inverted $U$-tube or $\Omega$-tube, which is completely sealed

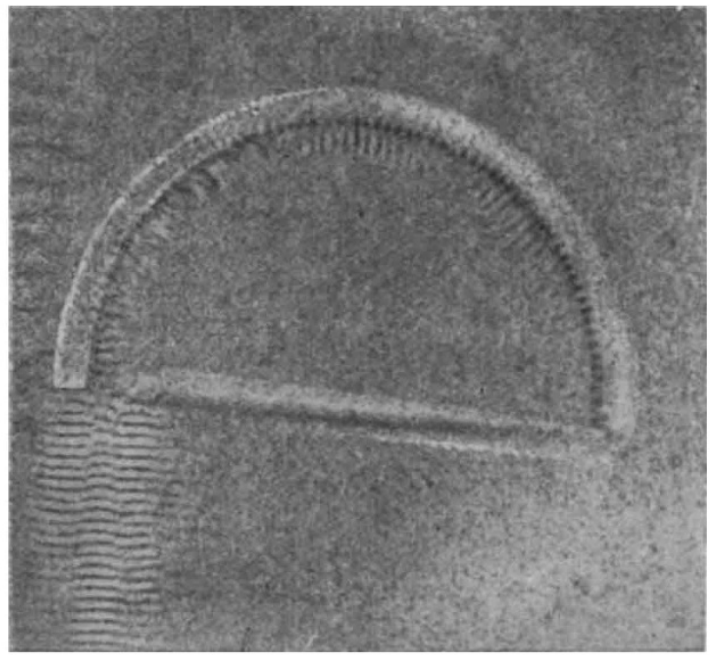

Fig. 3.--Model of lecture theatre of Royal Institution, showing ' whispering gallery ' effect, illustrated by stationary waves of ultrasonic sound of $2 \mathrm{~mm}$. wave-length. Notice how the waves hug the wall.

up and contains liquid in one limb only, we observe the rapid vaporisation of liquid in the agitated 
limb and its deposition as liquid in the previously empty limb. This might appropriately be called acoustic distillation.

On substituting for the inverted U-tube a similar tube which contains a little dry lycopodium powder

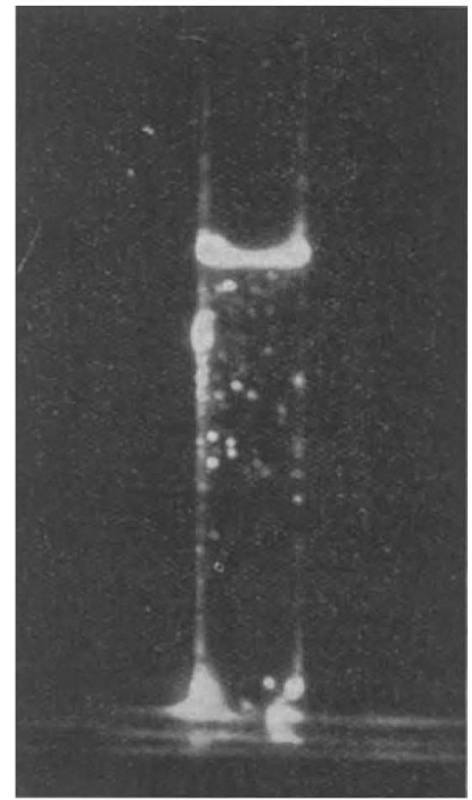

FiG. 4.--Expulsion from water of dissolved air as bubbles.

but is otherwise highly evacuated, an astonishing result is obtained. The powder sometimes moves in this case towards the limb which is subjected to the more intense vibration. This last effect is one of a number of phenomena due to the transverse vibrations of various types set up in the walls of the vessel itself. Another of these effects is the formation of ripples in the oil splashed on the tube and the repulsion of the oil drops away from the source of disturbance.

The most striking thing, however, about the oil drops is their behaviour at a constriction, or at the narrower parts of a tube such as is shown in Fig. 1. High inputs of ultrasonic energy cause intense local heating at such places, and the oil is thrown off as spray and jets of vapour as from diminutive geysers. If the tube is drawn off into a fine fibre and firmly grasped between the fingers, a severe burn is ex-

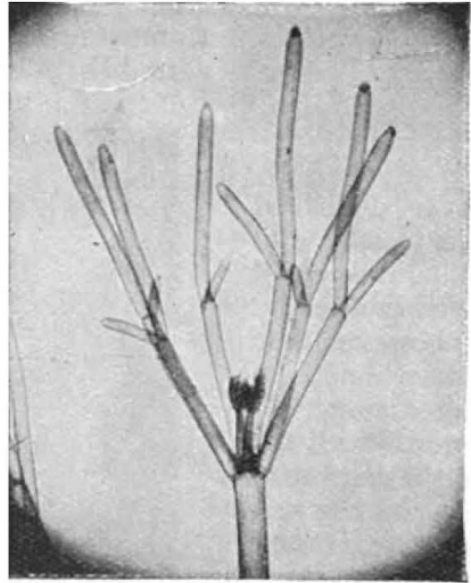

(a)
Most of the effects obtainable with the ultrasonic beam directed towards the surface of a liquid were discovered by Wood and Loomis in the United States. Acoustic distillation, the anomalous movement of lycopodium powder, and the non-liberation of bubbles from water with very high intensities are, we believe, described now for the first time.

\section{Biological Effects of Ultrasonics.}

Some fifteen years ago, while carrying out experiments at sea, Langevin and his co-workers observed that fish which swam into a beam of ultrasonic waves were disabled or killed. During the past few years I have been able to investigate this phenomenon. Quite independently, Wood and Loomis covered much the same ground. Let us notice first what happens to an object the dimensions of which are large compared with the wavelength of the sound waves which irradiate it. One result can be seen from Fig. 5. These are reproductions of photomicrographs of a freshwater plant -Nitella. The irradiated specimen shows how certain regions in a cell are denuded of their chloroplasts, which are then piled up in other spots. A gentler irradiation merely causes the streaming of the protoplasm to cease either temporarily or permanently according to the duration of exposure. The mechanical action which has taken place here can be understood when it is realised what occurs in the water in which the plant is immersed. $\mathrm{Al}$ though the excursion of a disturbed particle during a single vibration is only equal to a few wavelengths of light, these vibrations, occurring at the rate of nearly a million times a second, cause the maximum velocity of the particle to be very high ;

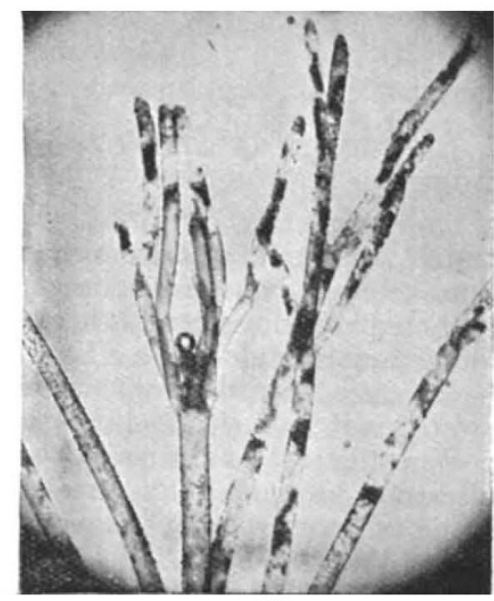

(b)
F1G. 5.-(a) Normal Nitella; (b) Nitella after exposure to ultrasonic vibrations. $(n=750,000$.) perienced. In addition to the calorific effects produced in the walls of various containers, the absorption of sound energy which occurs in their contents causes appreciable rises of temperature in them also. Amongst other effects which we can produce by our installation, we may cite the acceleration of certain chemical reactions, the flocculation of suspended matter, and the emulsification of oil and water, etc.

its maximum acceleration may become as great as $10 \mathrm{~km}$. per sec. per sec. We have, in effect, a micro ultra-centrifuge, which subjects specimens to very intense though highly localised forces.

This explanation accounts quite satisfactorily for certain other effects we have obtained, such as the breakdown of the red blood-corpuscle and the disruption of Planaria and similar small living organisms. The same explanation may possibly account 
for the decreased response to stimulation of an irradiated muscle-nerve preparation and the arrest or retardation of the beating of the isolated frog's heart and embryonic heart of a chick.

Further explanation is required to account for the action on bacteria and viruses-bodies which are of microscopic and ultra-microscopic dimensions. We have found that the agglutinating power of certain streptococci is increased after irradiation, but the virulence of a strain of pneumococcus was unchanged or changed in an indefinite manner. (Preliminary experiments with bacteriophage have given a negative result.)

Positive effects have been obtained with one virus. The accompanying table shows the effect of ultrasonic vibrations in increasing the potency of the virus of vaccinia. Through the kindness of Dr. Mervyn Gordon, the irradiated and control lymphs in various dilutions were used to vaccinate the skin of a rabbit. The 'takes' were recorded after the requisite period of time had elapsed for the characteristic lesions to be produced. Two series of experiments were carried out, the lymph in the first case being given only one-fifth of the exposure given in the second case. It will be noticed that the result is the same in both cases. Presumably the effect of the exposure was merely to disengage inert foreign material from the virus so that the latter could exhibit its intrinsic potency.

Effect of Uttrasonic Vibrations on Vaccinia Virus. (Frequency 700,000.)

$\begin{array}{lccc}\text { Dilution. } & \text { 1st Expt. } & \text { 2nd Expt. } & \text { Control. } \\ 1 / 1 & + & + & + \\ 1 / 10 & + & + & + \\ 1 / 50 & + & + & + \\ 1 / 100 & + & + & + \\ 1 / 500 & + & + & - \\ 1 / 1000 & + & + & - \\ 1 / 2000 & - & - & -\end{array}$

We have seen that fish may be killed by means of inaudible sound waves. With so many effects occurring simultaneously-the breaking up of blood corpuscles; liberation of dissolved gases ; paralysis of muscle and nerve; localised mechanical action and heat production-this should no longer surprise us. In conclusion, it may be added that ultrasonic radiation is not a menace to human life. The vibrations are of necessity produced in a liquid because at these high frequencies the waves are so rapidly attenuated in air that their propagation through it is practically impossible.

\section{Obituary.}

Dr. Thos. A. Edison.

BY the death at Llewellyn Park, West Orange, New Jersey, on Oct. 18, of Thomas Alva Edison, America has lost one of its most famous men, and one whose name, like those of his countrymen Fulton, Whitney, Colt, Morse, Bell, Maxim, Westinghouse, and the Wright brothers, will always be remembered as that of a great inventor. When a youth, Edison bought a set of Faraday's works, and he afterwards said, "I think I must have tried everything in those books." It was in this direction his great strength lay; for, gifted with a vivid imagination and a quick and active mind, he possessed a remarkable willingness to put everything to the test, and to seek in any and every direction for a solution to the problem of the moment.

Edison came of Dutch and Scotch descent, and was born at Milan, Ohio, on Feb. 11, 1847. It was apparently from his mother that he inherited his intense mental activity, while from his father's side came his exceptional physique and powers of endurance. His great-grandfather lived to the age of a hundred and four years, his grandfather to a hundred and two, and his father and several uncles to more than ninety years of age. The romantic story of his early life has many parallels in Ameriean biography. Owing nothing to schools or masters, he was his own instructor and the founder of his own fortunes. As a train boy of fifteen years of age he printed a small newspaper, the first ever produced on a train in motion; at sixteen years he was a telegraph operator; at nineteen he took out his first patent; at twenty-two he was working for a telegraph company at 300 dollars a month, and soon afterwards was able to sell his improvements in 'stock tickers' for 40,000 dollars, and in 1876 to open his laboratory at Menlo Park, Newark, New Jersey.

The record of the work of Edison and his assistants during the next twenty years at Menlo Park and at West Orange, to which he removed in 1887 , probably has no parallel in the history of invention, and it is no wonder he became popularly known as the 'Wizard of Menlo Park'. It has been said of him that " he could always be counted upon to play his part in the mechanical evolution of new inventive arts ", but his part often became the principal one. In telegraphy he will be remembered for the invention in 1872 of the type printing receiver and his subsequent work on quadruplex telegraphy, while to telephony he contributed the important invention of the carbon transmitter. Bell invented the telephone in 1876, Hughes the microphone in 1878 , and Edison the carbon transmitter in 1877. To that year also belongs his outstanding invention of the phonograph.

Two years later Edison successfully solved the problem of "the subdivision of the electric light" by the invention of his incandescent lamp, his work in this direction being contemporary with that of Maxim, Lane Fox, and Swan. His improvements in the dynamo, made about the same time, gave him a place beside Gramme, Siemens, Crompton, Brush, and Hopkinson; while the first central power stations in both the Old World and the New World, the former in Holborn Viaduct, London, and the latter in Pearl Street, New York, were the direct outcome of his many inventions. Both these stations began operations in $\mathbf{1 8 8 2}$.

In Menlo Park, in 1880, Edison made experiments with electric traction, while he afterwards 